\title{
Papillomes et cancers cholédociens
}

II est toujours troublant d'apprendre qu'une affection considérée comme exceptionnelle, est rencontrée avec une frequence non négligeable dans un certain service medical: J. Carolí peut faire état de 12 observations de tumeurs papillomateuses du cholédoque, alors qu'il n'en existait que 25 cas publiés depuis 1894 ! L'étude approfondie qu'il y consacre avec ses collaborateurs chirurgiens (J. Carolí; R. Soupault; M. Champeau; J. Etévé et M. Hívet: Revue des Maladies du Foie 4: 191-231 [1959]) mérite d'etre signalée, car elle représente une mise au point parfaite d'un sujet trop méconnu, même des spécialistes.

Les papillomes obstructífs des voies biliaires siègent de preference sur le bas cholédoque, la papille de Vater étant prédisposée aux productions hyperplasiques. Cliniquement, l'affection est trompeuse, car ces tumeurs peuvent entraîner des ictères mécaniques brutaux ou insidieux (simulant le cancer de la tête pancréatique) ou des syndromes pseudo-lithiasiques. Le diagnostic per-opératoire de nature est capital: il n'y a aucun rapport entre la taille de la tumeur et sa malignité éventuelle; en revanche, sa consistance molle doit faire préjuger de sa bénignité. Cette notion est essentielle pour diriger l'acte chirurgical: l'exérèse locale ou le curettage simple sont suffisants pour combattre les papillomes bénins.

Mais Гarbre biliaire tout entier peut être atteint à^hyperplasíe vílleuse qui s’exprime par des écoulements de mucus et de sang capables d'entraîner de Гanémie et des obstructions intermittentes de la voie biliaire principale. Le syndrome clinique est grave, l'acte chirurgical le plus souvent impuissant à rétablir l'écoulement biliaire.

*

A ces tumeurs bénignes, il faut opposer le cancer de la voie biliaire príncípale, qui est moins frequent encore que le cancer vésiculaire, et représente $1 \%$ seulement de tous les ictères. Ayant pu en recueillir 17 observations, J. P. Benhamou, A. Gottfried, F. Darnís et R. Fau• vert présentent une excellente revue d'ensemble dans la Semaíne des Hôpítaux de Paris (vol. 25: 89100 [1959]) de cette maladie excep-tionnellement grave, puisque la mort survient, en moyenne, 2 à 3 mois après l'apparition des premiers signes cliniques: asthénie, anorexie, 